\title{
Hiv-1 Gag Gene Mutations, Treatment Response and Drug Resistance to Protease Inhibitors: A Systematic Review and Meta-analysis Protocol
}

\author{
ALEX DURAND NKA \\ CIRCB
}

Georges Teto

CIRCB

\section{Maria-Mercedes Santoro \\ University of Rome Tor Vergata}

Valantine Ngum Ndze

Faculty of Medicine and Biomedical Sciences, University of Yaoundé 1

Désiré Takou

CIRCB

\section{Beatrice Dambaya}

CIRCB

Ezechiel Ngoufack Jagni Semengue

CIRCB

Lavinia Fabeni

Lazzaro Spallanzani National institute for Infectious Diseases

\section{Carlo-Federico Perno}

Univesity of Milan

Vittorio Colizzi

University of Rome Tor vergata

Francesca Ceccherini-Silberstein

University of Rome Tor vergata

Joseph Fokam ( $\square$ josephfokam@gmail.com )

CIRCB

\section{Protocol}

Keywords: HIV, Gag mutations, resistance, protease inhibitors

Posted Date: June 10th, 2020

DOI: https://doi.org/10.21203/rs.3.rs-34217/v1 
License: (c) (i) This work is licensed under a Creative Commons Attribution 4.0 International License. Read Full License 


\section{Abstract}

Background: Some mutations in the HIV-1 Gag gene are known to confer resistance to ritonavir-boosted protease inhibitors $(\mathrm{Pl} / \mathrm{r})$, but their clinical implications remain controversial and most genotyping algorithms only consider the protease gene to evaluate resistance to $\mathrm{Pl} / \mathrm{r}$. Thus, a thorough analysis of existing findings would provide a better understanding on the role of HIV-1 Gag gene on the resistance to $\mathrm{Pl} / \mathrm{r}$, by characterizing genotypic co-variations between Gag-mutations/polymorphisms and mutations of the protease region.

Methods: We will include randomized and non-randomized trials, cohort and cross-sectional studies published in English and French (from 2012 onwards), and relevant conference reports on PI/r HIV-1 Gag gene resistance associated mutations. Hand searching of the reference lists of relevant reviews will be conducted and we will also look for the aforementioned conference abstracts. PUBMED, EMBASE, CENTRAL, CINAHL databases will be used to conduct the searches. We will include studies of adults and/or children exposed to $\mathrm{Pl} / \mathrm{r}$ based therapies; and we will exclude studies of patients with specific comorbidities such as tuberculosis or opportunistic infections. Primary outcomes will be "the rate of viral suppression", "the proportion of patients with immune recovery" and "the level of drug resistance" on protease PI/r-based regimens. Secondary outcomes will be "HIV-1 Gag gene non polymorphic mutations according to $\mathrm{Pl} / \mathrm{r}$ based-regimens" and "viral subtypes/recombinants". Two reviewers will independently screen titles and abstracts, assess the full texts for eligibility and extract data. If data permits, random effects models will be used where appropriate. Subgroup and additional analyses will be conducted to explore the potential sources of heterogeneity.

Discussion: This study will help to better understand the implications of the Gag gene mutations of $\mathrm{Pl} / \mathrm{r}$-based ART response for HIV positive individuals, for a potential consideration in algorithms interpreting HIV drug resistance for clinical management.

\section{Prospero registration number: CRD42019114851}

\section{Introduction}

Many HIV patients have access to combined antiretroviral therapy (ART). Antiretroviral therapy generally works well to keep the virus suppressed and the patient healthy when the patient has good adherence [1]. However, the treatment only works as long as the virus is not resistant to the drugs used. When the first antiretrovirals were commercialized in 1980, drug resistance was a definite result for all patients and the duration of successful treatment was limited[1]. Nowadays, some patients are treated for many years without any resistance problem, while for others, drug resistance is a serious threat to their health[1].

The role of HIV protease is to cleave the polyprotein precursors Gag and Gag-pol at the specific sites called "cleavage site" during the virion maturation stage. This processing step is necessary for viral 
infectivity, and protease inhibition or genetic inactivation results in noninfectious viral particles, consequently, particle maturation is an excellent drug target as shown by inhibitors specifically targeting the viral protease (protease inhibitors) [2] [3].

Protease inhibitors (PIs) are among the most active antiretrovirals currently used to treat HIV infection. These compounds, which mimic the natural Gag and Gag-Pol substrates of HIV protease, inhibit the proteolytic activity of the enzyme and exert a potent inhibitory effect on HIV replication in vitro and in vivo [4-6].

Mechanically, the resistance of HIV enzymes to drug targeting is based on mutations in the respective gene, resulting in structural alterations of the target of the viral drug. Despite this apparently strict correspondence, cases of clinical failure under PI treatment have been reported in which changes in the protease (PR) gene do not provide sufficient explanation [7]. In addition to the amino acid changes in the viral enzyme, a unique feature of HIV resistance to Protease inhibitors is that resistance mutations occur not only in the protease itself - the direct target of the inhibitors, but also in some natural substrates of the protease the cleavage sites of Gag. These mutations enhance the interactions between the substrate and the mutated enzyme and increase the cleavage accordingly. Initially described as compensatory mutations capable of partially correcting the loss of viral capacity resulting from protease mutations, Gag modifications are now recognized as being directly involved in resistance $[8,9]$.

However, most current genotyping algorithms for $\mathrm{Pl} / \mathrm{r}$ treatment failure still consider the protease gene to predict resistance to anti-HIV drugs [10] . Here, we will examine the evidence that HIV-1 Gag gene mutations are an important component of HIV resistance to $\mathrm{Pl} / \mathrm{r}$ by addressing in detail all mutations in Gag that are selected under $\mathrm{PI} / \mathrm{r}$ pressure and how polymorphisms and mutations of $G a g$ affect $\mathrm{Pl} / \mathrm{r}$ therapy, an aspect of $\mathrm{PI} / \mathrm{r}$ resistance that is currently not included in genotypic $\mathrm{Pl} / \mathrm{r}$ resistance tests.

\section{Aim of the study}

The objective of our study is to review the literature for qualitative evidence for HIV-1 Gag gene mutations that confer resistance to protease inhibitors and how these mutations vary genetically. Our secondary aim will be to generate the covariance of protease mutations and Gag mutations to better understand the evolution of protease inhibitors treatment failure.

\section{Methods And Analysis}

The present protocol has been registered within the PROSPERO database (registration number CRD42019114851) and is being reported in accordance with the reporting guidance provided in the Preferred Reporting Items for Systematic Reviews and Meta-Analyses Protocols (PRISMA-P) statement [11] (Additional file 1). PRISMA focuses on ways in which authors can ensure the transparent and complete reporting of systematic reviews and meta-analyses. 


\section{Eligibility criteria}

\section{Design and Setting of the study}

Type of studies to be included. We will include randomized and non-randomized trials, cohort and crosssectional studies, evaluating HIV-1 Gag gene resistance-associated mutations and treatment outcomes in HIV positive patients under protease inhibitors containing regimens.

\section{Characteristics of the participants}

Participants. We will include studies of the general population living with HIV, both children and adults who are $\mathrm{PI} / \mathrm{r}$-experienced. Studies in patients with HIV-2 will not include and if HIV type is not specified, we would contact the study authors and request this missing information. The study would be listed as 'awaiting classification' while we wait for a response. We will exclude studies of patients with specific comorbidities such as tuberculosis or opportunistic infections.

Intervention: $\mathrm{Pl} / \mathrm{r}$-based regimens will be our intervention of interest. Studies focusing on patients under Atazanavir (ATV/r), Lopinavir (LPV/r) or Darunavir (DVR/r) based regimens will be considered as our group of interest.

Comparators: Given that studies of $\mathrm{PI} / \mathrm{r}$ experienced patients will be the only ones included, HIV-1 viral subtype (subtype B and non B) will serve as comparators.

Outcomes. Primary outcomes will be "the rate of viral suppression", "the proportion of patients with immune recovery", and "the level of drug resistance" on protease inhibitor-based regimens. Secondary outcomes will be "HIV-1 Gag gene non polymorphic mutations according to $\mathrm{Pl} / \mathrm{r}$ based-regimens" and "viral subtypes/recombinants".

Viral suppression is defined as plasma viral load less than 1000 copies of viral RNA. Level of resistance refers to the proportion of patients having at least one major $\mathrm{PI} / \mathrm{r}$ resistance mutation. The immune recovery of a patient refers to the CD4 count $>500$ cells $/ \mathrm{mm}^{3}$ following treatment according to individual studies. HIV-1 Gag non polymorphic mutations will be defined as mutations significantly associated with $\mathrm{PI} / \mathrm{r}$ exposure; Subtypes or recombinants refer to the viral strains identified in a patient sample.

Report characteristics: We will restrict the search to articles published in English and French, from 2012 onwards without geographical limitation.

\section{Information sources and search strategy}

We will conduct a comprehensive literature search with the help of two librarians with expertise in systematic reviews. 
Electronic databases. We will perform the searches in PubMed/MEDLINE, Embase, Cochrane Central Register of Controlled Trials (CENTRAL), Latin American and Caribbean Health Sciences Literature (LILAC) Web of Science, African Journals Online and Cumulative Index to Nursing and Allied Health Literature (CINAHL).

Trial registers: Ongoing trials will be sought in the WHO International Clinical Trials Registry Platform (ICTRP) and ClinicalTrials.gov.

Conference abstracts. We will search conference abstract archives on the websites of the Conference on Retroviruses and Opportunistic Infections (CROI); the International AIDS Conference (IAC); the International AIDS Society Conference on HIV Pathogenesis, Treatment, and Prevention (IAS) and all Virology Education conferences, for all available abstracts presented at all conferences from January 2012 onwards.

Other sources. Hand searching of the reference lists of relevant reviews and trials will be conducted. In addition, we will contact experts in the field for other potentially eligible studies we may have missed.

The Medical Subject Headings (MeSH terms) for HIV and AIDS and key terms "Gag mutations", "Protease inhibitors", "drug resistance" 'will be cross-referenced (Additional file 2 shows the detailed search strategy for Pubmed, Embase and CINAHL). We will update the search prior to publication to include any additional eligible papers published recently (Additional file 2).

\section{Study records}

\section{Data management}

All records from the various sources included in our search strategy will be combined, uploaded into the reference management software Zotero ${ }^{\circledR}$ (version 5.0.85) and de-duplicated. We will use Microsoft Excel (version 2016 for Windows, Microsoft Corp., Redmond, WA, USA) to record outcomes of the selection process.

\section{Selection of eligible studies}

Two reviewers (ADN and GT) will independently screen the titles and abstract to identify potentially eligible studies using an eligibility form. Disagreements will be resolved by consensus, if necessary through discussion with a third reviewer (DT or ENJS). The full texts of potentially eligible articles will be obtained and independently reviewed by two authors (ADN and GT) to identify included studies.

Discrepancies will be resolved by consensus and if necessary through discussion with a third reviewer (JF or DT). Studies that are being conducted at the time of the review and which do not yet have results will be identified as ongoing. And studies that have been completed without results could be classified as 
studies awaiting classification excluded studies and their reasons for exclusion will be described. The PRISMA [11] study flow diagram will reflect this process and detail the reasons for exclusion of studies.

\section{Data collection}

We will develop a data extraction sheet to guide data extraction. The sheet will be pilot-tested by two reviewers (VNN and JF) on a random sample of 05 articles and revised as needed. Two reviewers will independently read each eligible full text article and extract the relevant data. Both sets of data will be entered into Microsoft Excel (version 2016 for Windows, Microsoft Corp., Redmond, WA, USA). Any discrepancies in the extracted data will be resolved by consensus, in discussion with a third reviewer (DT or JF) if necessary.

\section{Study selection process}

Two reviewers will independently select studies at two levels: title / abstract, followed by full text analysis. A checklist with the eligibility criteria will be developed and tested on a random sample of 5 studies. Titles and abstracts will then be compared to eligibility criteria. Two reviewers will independently apply the inclusion criteria to the complete texts. At both stages of selection, we will resolve disagreements by consensus, if necessary, through discussion with a third reviewer. We will record any discrepancies on Excel spreadsheets, with reasons for inclusion or exclusion. The PRISMA study flow diagram will reflect this process and detail the reasons for exclusion of studies.

\section{Data items}

We will extract the following from included studies:

- Study characteristics (year of publication, study period, study population, study design, aim of study, geographic location, duration of follow-up);

- Study setting (location and type of facilities);

- Characteristics of study population (sample size, age, sex, marital status, weight, enrolment period, inclusion and exclusion criteria);

- $\mathrm{Pl} / \mathrm{r}$-based regimens;

- Duration of $\mathrm{Pl} / \mathrm{r}$-based regimens;

- Viral suppression;

- CD4-count following treatment;

- Protease major and minor resistant mutations ;

- Gag gene non polymorphic mutations;

- HIV-1 subtypes;

There is no pre-planned data assumption. 


\section{Data Synthesis}

The main characteristics of all included studies will first be narratively synthesized (i.e. year of publication, study period, study population, study design, aim of study, geographic location, duration of follow-up). A descriptive analysis of study characteristics will be undertaken to explore the heterogeneity of the studies. Summary statistics will then be used to describe study outcomes, including means or medians, and frequencies. Proportions with exact binomial 95\% confidence intervals ( $95 \% \mathrm{Cl}$ ) will be calculated for each outcome and presented in forest plots. We will calculate the between-study variance (tau-squared) and p-values from tests of between-study heterogeneity. We expect substantial betweenstudy heterogeneity, and the focus of the subsequent analyses will therefore be on the identification and exploration of sources of heterogeneity. Finally, we will explore associations that may exist between proportions in countries, and study outcomes (i.e. the rate of viral suppression and the level of drug resistance) using random intercept logistic meta-regression (Binomial-Normal) models. These models avoid the biases that arise when Normal-Normal models are applied to log it or arcsine-square root transformed proportions. Where appropriate, we will use the same models to calculate combined estimates of proportions. We will use the GRADE approach to rate the certainty of evidences as "high", "moderate", "low" and "very low [12]. Major findings on Gag gene non polymorphic mutations, protease mutations, viral subtype, drug susceptibility and linkage to $\mathrm{Pl} / \mathrm{r}$ (ATV/r, LPV/r, and DRV/r) will be summarized in a table. This table will also present the quality of the evidence found, all sorted according to socio-demographic data, clinical and laboratory parameters. Though the highest quality rating is for randomized trial evidences, they may be downgraded to moderate, low, or even very low quality evidence. Rating will depend on limitations in study design and implementation, indirectness of evidences, unexplained heterogeneity, imprecision of result and a high probability of publication bias[12]. However, if such studies yield large effects and there is no obvious bias explaining those effects, we would rate the evidences as moderate or - if the effect is large enough - even high quality [12] (See Additional file 3).

\section{Additional analysis}

Subgroup and additional analyses will be conducted following stratification of the study participants. Results will then be sorted according to age (adults/adolescents vs. children [ 10 years]), sex (male vs. female), viremia, level of CD4 following treatment, $\mathrm{Pl} / \mathrm{r}$-based regimens, adherence level, and adverse reactions. This will allow us to adjust for potential confounders, to better estimate the effect of each variable on the observed outcomes. If data permits, meta-regression will be performed and summary estimates will help explore the relationship between study-covariates and effect size, in order to highlight any statistical significance.

\section{Dealing with missing data}

If data are missing in key variables, we will contact the study authors for clarifications. A description of missing data will be provided for each study, and we will discuss the possible implications of missing data. 


\section{Assessment of the risk of bias in individual studies}

Two reviewers (ADN and ENJS) will assess eligible studies using ROBINS-I [13], a tool for assessing risk of bias in non-randomized studies of interventions. ROBINS provides a systematic way to organize and present the available evidence related to risk of bias in these studies. ROBIS [RoB 2.0] [14] will be used in parallel for randomized studies, which include randomized controlled trials. Risks of bias in cohort and case-control studies will be assessed using the Newcastle-Ottawa Scale [15]. These tools will help assess studies but not their outcomes, and will be adapted to the context of this systematic review.

\section{Statistical software}

All analyses will be done in R version 3.6.1 and Microsoft Excel version 2016 (Windows, Microsoft Corp., Redmond, WA, USA). R will help us calculate means, medians, frequencies, percentages, confidence intervals and assess primary associations between variables using statistical tests. We will use a validated Excel spreadsheet for meta-analysis and Forest plots (https://www.ncbi.nlm.nih.gov/pmc/articles/PMC3296675/), as previously described [16].

\section{Ethics and dissemination:}

As this study will be based on published data, ethical approval is not required. The final report will be disseminated through publication in a peer reviewed scientific journal and will also be presented at relevant conferences.

\section{Discussion}

This study will help to better understand protease inhibitors treatment failure in HIV positive individuals by demonstrating the involvement of Gag gene mutations in this resistance process. This study will also shed light on the variation of mutations in the Gag gene as a function of the distribution of the different subtypes of HIV-1 and highlight all the major mutations in the Gag gene involved in $\mathrm{Pl} / \mathrm{r}$ resistance and their covariance with the mutations present in the protease gene. This systematic review will also identify gaps encountered in these studies. Our results will be used to help virologists and physicians to better understand protease inhibitor failures without resistance mutations in the protease gene and to provide important arguments for a possible introduction of the Gag portion into the HIV resistance-testing panel. As potential limitations of this review, we may be confronted with important study heterogeneity and incompleteness, but these will be considered in statistic models during meta-regression analysis; if not perform, studies incompleteness at least would be solve by contacting study authors. Another limitation may be at the level of reviewing and including studies. In effect, in the process of resolving disagreements while reviewing articles, all team members will be included in the decision-making process or at least aware of the disagreements being discussed. We will try as much as possible to have a consensus decision for each disagreement. Important protocol amendments will be documented, taken into consideration while analyzing the data, and discussed consequently in the final paper. Our findings will be published in a peer-review journal and subsequently disseminated to policy-makers first at the 
national level through the submission of a governmental notice, and at the international level through conferences and stakeholder meetings.

\section{Abbreviations}

AIDS: Acquired immunodeficiency syndrome

ART: Antiretroviral therapy

CCTs: Clinical trials

CROI: Conference on Retroviruses and Opportunistic Infection

Gag: Group-specific antigen

Gag-Pol: Group-specific antigen and polymerase

HIV: Human immunodeficiency virus

ITS: Interrupted time series.

PRISMA-P: Preferred Reporting Items for Systematic Review and Meta-analysis Protocols

PROSPERO: International Prospective Register of Systematic Reviews

$\mathrm{PI} / \mathrm{r}$. Protease inhibitors boosted by ritonavir

ROBINS-I: Risk of Bias in Non-randomized Studies - of Interventions

\section{Declarations}

\section{Ethics approval and consent to participate}

Not applicable

\section{Consent for publication}

Not applicable

\section{Availability of data and materials}

Not applicable

\section{Competing interests}

Authors have no potential conflict or competing interests to declare.

\section{Funding}

No funding has been received for this protocol.

\section{Authors' contributions}


Conception and design of the review: Alex Durand Nka; Georges Teto; Joseph Fokam; Valantine Ngum Ndze; Maria Mercedes Santoro; Vittorio Colizzi; Carlo Federico Perno.

Developed the search strategies: Alex Durand Nka; Valantine Ngum Ndze ; Joseph Fokam; Georges Teto; Desire Takou; Ezechiel Ngoufack Jagni Semengue; Beatrice Dambaya.

Drafted the manuscript: Alex Durand Nka; Valantine Ngum Ndze; Joseph Fokam; Georges Teto; Ezechiel Ngoufack Jagni Semengue.

Involved in critically revising the protocol for important intellectual content: Alex Durand Nka; Joseph Fokam; Valantine Ngum Ndze.

\section{Approved the final version of the submitted manuscript: All the authors.}

\section{Acknowledgements}

We thank the "Chantal BIYA" International Reference Centre to hosting the Work sessions of drafting the Systematic Review protocol

\section{References}

1. Pennings PS. HIV Drug Resistance: Problems and Perspectives. Infect Dis Rep [Internet]. 6 juin 2013 [cité 13 déc 2018];5(Suppl 1). Disponible sur: https://www.ncbi.nlm.nih.gov/pmc/articles/PMC3892620/

2. Hilton BJ, Wolkowicz R. An Assay to Monitor HIV-1 Protease Activity for the Identification of Novel Inhibitors in T-Cells. PLoS One [Internet]. 3 juin 2010 [cité 13 déc 2018];5(6). Disponible sur: https://www.ncbi.nlm.nih.gov/pmc/articles/PMC2880603/

3. Pettit SC, Moody MD, Wehbie RS, Kaplan AH, Nantermet PV, Klein CA, et al. The p2 domain of human immunodeficiency virus type $1 \mathrm{Gag}$ regulates sequential proteolytic processing and is required to produce fully infectious virions. J Virol. déc 1994;68(12):8017-27.

4. Clavel F, Mammano F. Role of Gag in HIV Resistance to Protease Inhibitors. Viruses. 5 juill 2010;2(7):1411-26.

5. Weber IT, Kneller DW, Wong-Sam A. Highly resistant HIV-1 proteases and strategies for their inhibition. Future Med Chem. juin 2015;7(8):1023-38.

6. Arts EJ, Hazuda DJ. HIV-1 Antiretroviral Drug Therapy. Cold Spring Harb Perspect Med. 4 janv 2012;2(4):a007161. 
7. De Luca A. The impact of resistance on viral fitness and its clinical implications. In: Geretti AM, éditeur. Antiretroviral Resistance in Clinical Practice [Internet]. London: Mediscript; 2006 [cité 14 déc 2018]. Disponible sur: http://www.ncbi.nlm.nih.gov/books/NBK2244/

8. Dam E, Quercia R, Glass B, Descamps D, Launay O, Duval X, et al. Gag Mutations Strongly Contribute to HIV-1 Resistance to Protease Inhibitors in Highly Drug-Experienced Patients besides Compensating for Fitness Loss. PLoS Pathog [Internet]. 20 mars 2009 [cité 12 déc 2018];5(3). Disponible sur: https://www.ncbi.nlm.nih.gov/pmc/articles/PMC2652074/

9. Mechanisms of HIV Drug Resistance [Internet]. [cité 14 déc 2018]. Disponible sur: https://www.prn.org/index.php/management/article/hiv_drug_resistance_mechanisms_277

10. Shafer RW. Genotypic Testing for Human Immunodeficiency Virus Type 1 Drug Resistance. Clin Microbiol Rev. avr 2002;15(2):247-77.

11. Moher D, Shamseer L, Clarke M, Ghersi D, Liberati A, Petticrew M, et al. Preferred reporting items for systematic review and meta-analysis protocols (PRISMA-P) 2015 statement. Syst Rev. 1 janv 2015;4:1.

12. Higgins J, Sally G. Cochrane Handbook for Systematic Reviews of Interventions. The Cochrane Collaboration, éditeur. John Wiley \& Sons Ltd, The Atrium, Southern Gate, Chichester, West Sussex P019 8SQ, England Telephone (+44) 1243 779777; 2008. 674 p.

13. Sterne JA, Hernán MA, Reeves BC, Savović J, Berkman ND, Viswanathan M, et al. ROBINS-l: a tool for assessing risk of bias in non-randomised studies of interventions. BMJ. 12 oct 2016;355:i4919.

14. Whiting P, Savović J, Higgins JPT, Caldwell DM, Reeves BC, Shea B, et al. [ROBIS: a new tool to assess risk of bias in systematic reviews was developed.]. Recenti Prog Med. sept 2018;109(9):421-31.

15. Risk of Bias in Cohort Studies [Internet]. Systematic Review and Literature Review Software by Evidence Partners. [cité 18 avr 2020]. Disponible sur: https://www.evidencepartners.com/resources/methodological-resources/risk-of-bias-in-cohortstudies/

16. Neyeloff JL, Fuchs SC, Moreira LB. Meta-analyses and Forest plots using a microsoft excel spreadsheet: step-by-step guide focusing on descriptive data analysis. BMC Res Notes. 20 janv 2012; $5: 52$.

\section{Supplementary Files}

This is a list of supplementary files associated with this preprint. Click to download.

- Additionalfile3.DOCX

- Additionalfile2.DOCX

- BMCSystRevCoverLetterHIVGagfinal1.docx

- AdditionalFile1.docx 